\title{
Keplerian frequency of uniformly rotating neutron stars and strange stars
}

\author{
P. Haensel ${ }^{1}$, J. L. Zdunik ${ }^{1}$, M. Bejger ${ }^{1}$, and J. M. Lattimer ${ }^{2}$ \\ 1 N. Copernicus Astronomical Center, Polish Academy of Sciences, Bartycka 18, 00-716 Warszawa, Poland \\ e-mail: [haensel; jlz; bejger] acamk.edu.pl \\ 2 Department of Physics and Astronomy, State University of New York at Stony Brook, Stony Brook, NY 11794-3800, USA \\ e-mail: lattimer@astro.sunysb.edu
}

Received 30 December 2008 / Accepted 30 April 2009

\begin{abstract}
Aims. We calculate Keplerian (mass shedding) configurations of rigidly rotating neutron stars and strange stars with crusts. We check the validity of the empirical formula for Keplerian frequency, $f_{\mathrm{K}}$, proposed by Lattimer \& Prakash, $f_{\mathrm{K}}(M)=$ $C\left(M / M_{\odot}\right)^{1 / 2}(R / 10 \mathrm{~km})^{-3 / 2}$, where $M$ is the (gravitational) mass of the Keplerian configuration, $R$ is the (circumferential) radius of the non-rotating configuration of the same gravitational mass, and $C=1.04 \mathrm{kHz}$.

Methods. Numerical calculations are performed using precise 2D codes based on the multi-domain spectral methods. We use a representative set of equations of state (EOSs) of neutron stars and quark stars.

Results. We show that the empirical formula for $f_{\mathrm{K}}(M)$ holds within a few percent for neutron stars with realistic EOSs, provided $0.5 M_{\odot}<M<0.9 M_{\max }^{\text {stat }}$, where $M_{\max }^{\text {stat }}$ is the maximum allowable mass of non-rotating neutron stars for an EOS, and $C=C_{\mathrm{NS}}=1.08 \mathrm{kHz}$. Similar precision is obtained for strange stars with $0.5 M_{\odot}<M<0.9 M_{\max }^{\text {stat }}$. For maximal crust masses we obtain $C_{\mathrm{SS}}=1.15 \mathrm{kHz}$, and the value of $C_{\mathrm{SS}}$ is not very sensitive to the crust mass. All our $C$ s are significantly larger than the analytic value from the relativistic Roche model, $C_{\text {Roche }}=1.00 \mathrm{kHz}$. For $0.5 M_{\odot}<M<0.9 M_{\max }^{\text {stat }}$, the equatorial radius of the Keplerian configuration of mass $M, R_{\mathrm{K}}(M)$, is, to a very good approximation, proportional to the radius of the non-rotating star of the same mass, $R_{\mathrm{K}}(M)=a R(M)$, with $a_{\mathrm{NS}} \approx a_{\mathrm{SS}} \approx 1.44$. The value of $a_{\mathrm{SS}}$ is very weakly dependent on the mass of the crust of the strange star. Both $a$ values are smaller than the analytic value $a_{\text {Roche }}=1.5$ from the relativistic Roche model.
\end{abstract}

Key words. dense matter - equation of state - stars: neutron - stars: rotation

\section{Introduction}

Because of their strong gravity, neutron stars can be very rapid rotators. In view of the high stability of pulsar frequency (even the giant glitches produce relatively small fractional changes of rotation frequency, $\lesssim 10^{-5}$ ), one can treat pulsar rotation as rigid. The frequency $f$ of stable rotation of a star of gravitational mass $M$ and baryon mass lower than the maximum allowable for non-rotating stars is limited by the (Keplerian) frequency $f_{\mathrm{K}}$ of a test particle co-rotating on an orbit at the stellar equator. The relation between $f_{\mathrm{K}}$ and stellar gravitational mass $M$, $f_{\mathrm{K}}=f_{\mathrm{K}}(M)$, depends on the (unknown) equation of state (EOS) at supranuclear densities. Both quantities, $M$ and $f$, are measurable, and the condition implied by a measured frequency $f_{\text {obs }}$ of a pulsar of mass $M, f_{\mathrm{obs}}<f_{\mathrm{K}}(M)$, could be used to constrain theoretical models of dense matter (for a recent review of the theory of dense matter see Lattimer \& Prakash 2006; Haensel et al. 2007). Reliable determination of $f_{\mathrm{K}}(M)$ requires precise $2 \mathrm{D}$ calculations of stationary, extremely rapid rotating configurations in general relativity. Therefore, the search for a sufficiently precise, approximate but universal formula for $f_{\mathrm{K}}(M)$ is of great interest. Lattimer \& Prakash (2004) proposed an approximate empirical formula $f_{\mathrm{K}}(M) \approx C\left(M / M_{\odot}\right)^{1 / 2}(R / 10 \mathrm{~km})^{-3 / 2}$, where $R=R(M)$ is the circumferential radius of static star of mass $M$, and $C=1.04 \mathrm{kHz}$ does not depend on the EOS. In the present paper we calculate precise $2 \mathrm{D}$ models of rapidly rotating neutron stars and quark stars with different EOSs. We use the relativistic Roche model (Shapiro et al. 1983) to motivate the empirical formula for $f_{\mathrm{K}}(M)$ proposed by Lattimer \& Prakash (2004). We calculate the optimal value of prefactor $C$ and we establish limits for the validity of the empirical formula.

As of this writing, the maximum rotation frequency of a pulsar is $716 \mathrm{~Hz}$ (PSR J1748-2446ad, Hessels et al. 2006). Kaaret et al. (2007) reported the discovery of an oscillation frequency of $1122 \mathrm{~Hz}$ in an X-ray burst from the X-ray transient, XTE J1739-285, and concluded that "this oscillation frequency suggests that XTE J1739-285 contains the fastest rotating neutron star yet found", but this observation has not been confirmed or reproduced.

The problem of the constraint $f_{\max }^{\mathrm{EOS}}(M)>f_{\text {obs }}$ was considered by Shapiro et al. (1983) after the discovery of the first millisecond pulsar, PSR 1937+214, with $f_{\text {obs }}=641 \mathrm{~Hz}$ (Backer et al. 1982). Shapiro et al. (1983) used a formula for $f_{\max }(M)$ based on the relativistic Roche model. After the announcement of the discovery of a $2 \mathrm{kHz}$ pulsar, this formula was used to show that nearly all EOSs of dense matter existing at that time were ruled out by this observation (Shapiro et al. 1989).

In Sect. 2, we summarize results obtained with the relativistic Roche model. Section 3 contains description of realistic EOSs of nuclear matter, Sect. 4 provides the assumptions and methods used to calculate rotating stellar models. In Sect. 5 we check the validity of the empirical formula against results of precise 2D calculations for nine realistic EOS for neutron stars. Hypothetical self-bound quark stars with normal crusts are 
considered in Sect. 6. Section 7 presents the static and rotating configurations in the mass-radius plane. In Sect. 8 we derive approximate relations between the circumferential radius of a static configuration and that of a Keplerian configuration of the same gravitational mass, for neutron stars and quark stars. A discussion of our results is presented in Sect. 9.

\section{Relativistic Roche model}

There is a useful model of neutron stars for which an analytic formula for $f_{\mathrm{K}}(M)$ can be obtained (Shapiro et al. 1983, 1989). It is a relativistic Roche model, in which the mass of the star is assumed to be strongly centrally condensed. Consider a continuous sequence of stationary configurations of constant gravitational mass $M$, and rotation frequencies ranging from zero to $f_{\mathrm{K}}$. Let the circumferential radius of the non-rotating configuration be $R$. Under the assumption of an extreme central mass condensation, Shapiro et al. $(1983,1989)$ found an equation satisfied by the coordinates of the stellar surface (Eq. (2) of Shapiro et al. 1989). In the special case of the stellar equator, this equation implies that for normal equilibrium configurations rotating uniformly at $f$, the equatorial circumferential radius $R_{\text {eq }}$ satisfies

$\frac{2 G M}{R_{\mathrm{eq}}}+(2 \pi f)^{2} R_{\mathrm{eq}}^{2}=\frac{2 G M}{R}$,

where the extreme central concentration of matter implies that gravitational mass can be treated as constant and equal to the static value $M$. The left-hand-side of Eq. (1) reaches a minimum at $R_{\mathrm{eq}}=\left(G M / 4 \pi^{2} f^{2}\right)^{1 / 3}$. For the solution to exist for a given $f$, the value of the left-hand-side at this minimum should not exceed $2 G M / R$, which implies a condition on $f$ (Shapiro et al. 1983, 1989),

$f \leq f_{\mathrm{K}}=\frac{1}{2 \pi}\left(\frac{2}{3}\right)^{3 / 2}\left(\frac{G M}{R^{3}}\right)^{1 / 2}$.

Therefore, the Keplerian frequency is

$f_{\mathrm{K}}^{\text {Roche }}(M)=1.00 \mathrm{kHz}\left(\frac{M}{M_{\odot}}\right)^{1 / 2}\left(\frac{R}{10 \mathrm{~km}}\right)^{-3 / 2}$.

As stated in Shapiro et al. (1989), "the Relativistic Roche model provides a surprisingly accurate estimate of the maximum rotation rate along constant-rest mass sequences" for many realistic EOSs.

It is easy to show that an additional relation between the Keplerian and static configurations can be obtained. Using Eq. (1), one obtains a formula expressing $R_{\text {eq }}$ for the Keplerian configuration, $R_{\mathrm{K}}$, in terms of $R$ for a static configuration of the same mass $M$,

$R_{\mathrm{K}}(M)=\frac{3}{2} R(M)$.

The formula for $f_{\mathrm{K}}(M)$, Eq. (2), and that for $R_{\mathrm{K}}(M)$ imply that $f_{\mathrm{K}}(M)$ is equal to the orbital frequency of a test particle orbiting at $r=R_{\mathrm{K}}$ in the Schwarzschild space-time around a point mass $M$ at $r=0$. An approximate equality $f_{\mathrm{K}}(M) \approx$ $f_{\text {orb }}^{\text {Schw. }}\left(M, R_{\mathrm{K}}\right)$ was shown to be valid within a few percent for normal neutron stars and quark stars (Bejger et al. 2007). This relation holds strictly for the relativistic Roche model,

$f_{\mathrm{K}}^{\text {Roche }}(M)=f_{\text {orb }}^{\text {Schw. }}\left(M, R_{\mathrm{K}}\right)=\frac{1}{2 \pi}\left(\frac{G M}{R_{\mathrm{K}}^{3}}\right)^{1 / 2}$.

\section{Realistic EOSs of hadronic matter}

In view of our high degree of ignorance concerning the EOS of dense hadronic matter at supranuclear densities $(\rho>3 \times$ $10^{14} \mathrm{~g} \mathrm{~cm}^{-3}$ ), it is common to consider a set of EOSs based on different dense matter theories (for reviews, see Haensel et al. 2007; Lattimer \& Prakash 2006). We used nine theoretical EOSs. These EOSs are listed in Table 1, where the basic information (label of the EOS, theory of dense matter, reference to the original paper) is also presented.

Five EOSs are based on realistic models involving only nucleons (FPS, BBB, DH, APR, GN3). The remaining EOSs are softened at high density either by the appearance of hyperons (GNH3, BGN1H1), or a phase transition to a kaon-condensed state (GMGS-Km, GMGS-Kp).

For GMGS-Km and GMGS-Kp models, the hadronic Lagrangian is the same. However, to obtain GMGS-Kp, one assumes that the phase transition takes place between two pure phases and is accompanied by a density jump, calculated using the Maxwell construction. The GMGS-Km EOS is obtained assuming that the transition occurs via a mixed state of two phases (Gibbs construction). A mixed state is energetically preferred when the surface tension between the two phases is below a certain critical value. As the value of the surface tension is very uncertain, we considered both cases.

In all EOSs models (except FPS), the core EOS was joined with the DH EOS of the crust (Douchin \& Haensel 2001). For the FPS model, the FPS EOS of the core was supplemented with the FPS crust EOS of Lorenz et al. (1993).

Our set of EOSs includes very different types of models. This is reflected by a large scatter of the maximum allowable masses of non-rotating stars, $1.45 M_{\odot} \lesssim M_{\max } \lesssim 2.21 M_{\odot}$, and a range of circumferential radii of non-rotating stars with $M=1.4 M_{\odot}$, $10.85 \mathrm{~km} \lesssim R_{1.4} \lesssim 14.22 \mathrm{~km}$ (Table 1$)$.

\section{Calculating stationary rotating configurations}

The stationary configurations of rigidly rotating neutron stars have been computed in full general relativity by solving the Einstein equations for stationary axisymmetric spacetime (see Bonazzola et al. 1993; Gourgoulhon et al. 1999, for the complete set of partial differential equations to be integrated). The numerical computations were performed using the rotstar code from the LORENE library (http://www. lorene.obspm. fr). The code implements a multi-domain spectral method introduced in Bonazzola et al. (1998). A description of the code can be found in Gourgoulhon et al. (1999). The accuracy of the calculations has been checked by evaluation of the GRV2 and GRV3 virial error indicators (Gourgoulhon \& Bonazzola 1994; Bonazzola \& Gourgoulhon 1994), which showed values lower than $\sim 10^{-5}$.

\section{Maximum frequencies for realistic EOSs of neutron stars}

Our aim is to approximately reproduce precisely calculated Keplerian frequencies using an empirical formula

$f_{\mathrm{K}}(M) \approx C\left(\frac{M}{M_{\odot}}\right)^{1 / 2}\left(\frac{R}{10 \mathrm{~km}}\right)^{-3 / 2}$

where $M$ is the gravitational mass of rotating star and $R$ is the radius of the non-rotating star of mass $M, R=R(M)$.

Let us first consider each EOS separately and find a parameter $C_{\mathrm{EOS}}$ for an empirical formula suitable for that EOS. 
Table 1. Equations of state of the neutron star core.

\begin{tabular}{ccccc}
\hline \hline EOS & Model & Ref. & $\begin{array}{c}M_{\text {max }}^{\text {stat }} \\
{\left[M_{\odot}\right]}\end{array}$ & $\begin{array}{c}R_{1.4} \\
{[\mathrm{~km}]}\end{array}$ \\
\hline FPS & $\begin{array}{c}\text { N, energy } \\
\text { density functional }\end{array}$ & $\mathrm{a}$ & 1.800 & 10.85 \\
\hline GN3 & $\begin{array}{c}\mathrm{N}, \text { relativistic } \\
\text { mean field }\end{array}$ & $\mathrm{b}$ & 2.134 & 14.22 \\
\hline DH & $\begin{array}{c}\mathrm{N}, \text { energy } \\
\text { density functional }\end{array}$ & $\mathrm{c}$ & 2.048 & 11.69 \\
\hline APR & $\begin{array}{c}\mathrm{N}, \text { variational } \\
\text { theory }\end{array}$ & $\mathrm{d}$ & 2.212 & 11.42 \\
\hline BGN1H1 & $\begin{array}{c}\text { NH, energy } \\
\text { density functional }\end{array}$ & $\mathrm{e}$ & 1.630 & 12.90 \\
\hline BBB & $\mathrm{N}$, Brueckner theory & $\mathrm{f}$ & 1.920 & 11.13 \\
\hline GNH3 & $\begin{array}{c}\text { NH, relativistic } \\
\text { mean field }\end{array}$ & $\mathrm{g}$ & 1.964 & 14.20 \\
\hline GMGS-Km & $\begin{array}{c}\mathrm{N}+\text { mixed } \\
\text { N-kaon condensed }\end{array}$ & $\mathrm{h}$ & 1.454 & 13.12 \\
\hline GMGS-Kp & $\begin{array}{c}\mathrm{N}+\text { pure } \\
\text { N-kaon condensed }\end{array}$ & $\mathrm{i}$ & 1.450 & 13.20 \\
\hline
\end{tabular}

$\mathrm{N}$ - nucleons and leptons. NH - nucleons, hyperons, and leptons. Exotic states of hadronic matter are indicated explicitly. Maximum allowable mass for non-rotating stars, $M_{\max }^{\text {stat }}$, and the circumferential radius of nonrotating stars of $1.4 M_{\odot}, R_{1.4}$, are given in last two columns, respectively. References for the EOSs: a - Pandharipande \& Ravenhall (1989); b Glendenning (1985); c - Douchin \& Haensel (2001); d - A18 Argonne NN potential with relativistic corrections and Urbana modified UIX ${ }^{*}$ NNN potential model, from Akmal et al. (1998); e - Balberg \& Gal (1997); f - Paris two-body NN potential and Urbana UIX three body NNN potential, from Baldo et al. (1997); g - Glendenning (1985); h, ikaon condensate models with $U_{K}^{\mathrm{lin}}=-125 \mathrm{MeV}$, Pons et al. (2000).

We found that the parameter $C_{\mathrm{EOS}}$ can be very well defined using the approximately linear segment of each $f_{\mathrm{K}}(M)$ curve (Figs. $1-2)$ in the mass range $\left(0.5 M_{\odot} \div 0.8 M_{\max }^{\text {stat }}\right)$. The values of $C_{\text {Eos }}$ were determined by least square fits and range from $1.07 \mathrm{kHz}$ (GN3) up to $1.09 \mathrm{kHz}$ (APR), with an rms deviation of the order of $0.5 \%$. These values of $C_{\mathrm{EOS}}$ are systematically larger than the value $1.04 \mathrm{kHz}$ proposed in Lattimer \& Prakash (2004), and significantly larger the value $1.00 \mathrm{kHz}$ from the Relativistic Roche model (Eq. (3). We defined the EOSindependent $C_{\mathrm{NS}}=1.08 \mathrm{kHz}$ to be used in Eq. (6) as the mean value of the $9 C_{\text {EOS }}$ coefficients for the EOSs of Table 1 .

We recommend the use of this value over a somewhat broader range of masses, $\left(0.5 M_{\odot} \div 0.9 M_{\text {max }}^{\text {stat }}\right)$. In Figs. $1-2$ we compare precisely calculated Keplerian frequencies with those given by the empirical formula, assuming $C_{\mathrm{NS}}=1.08 \mathrm{kHz}$. The precision of the empirical formula for $f_{\mathrm{K}}$ stays remarkably high for $0.5 M_{\odot}<M<0.9 M_{\max }^{\text {stat }}$. Relative deviations are typically within $2 \%$, with a largest deviation of at most $5 \%$ at the highest mass end for the GNH3 EOS.

\section{Strange stars}

The case of strange stars, built from self-bound quark matter, is different from that of ordinary neutron stars. Matter distribution within quark stars has a very low density contrast between the quark core edge and its center. We considered three EOSs of self-bound quark matter, based on the MIT Bag Model (Farhi \& Jaffe 1984; Zdunik 2000). Model parameters are given

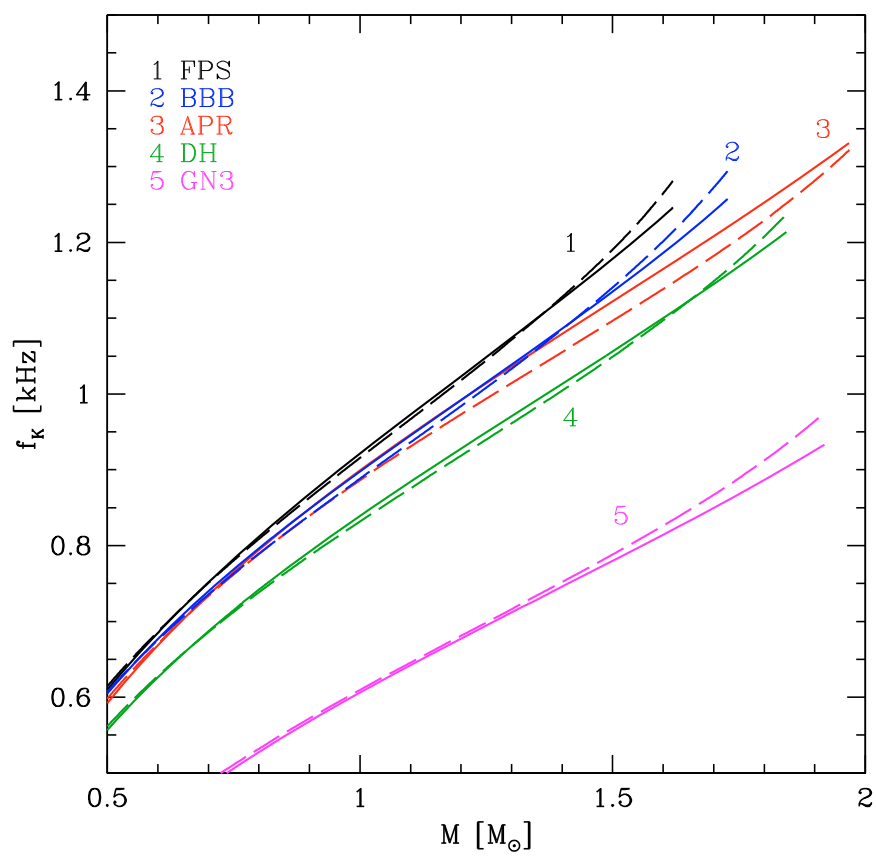

Fig. 1. (Color online) Precise values of Keplerian frequency $f_{\mathrm{K}}$ (solid line) and those calculated using Eq. (6) (dashed line), assuming $C=$ $C_{\mathrm{NS}}=1.08 \mathrm{kHz}$, versus stellar mass $M$. We consider masses $0.5 M_{\odot}<$ $M<0.9 M_{\max }^{\text {stat }}$ and APR, GN3, BBB, DH, and FPS EOSs.

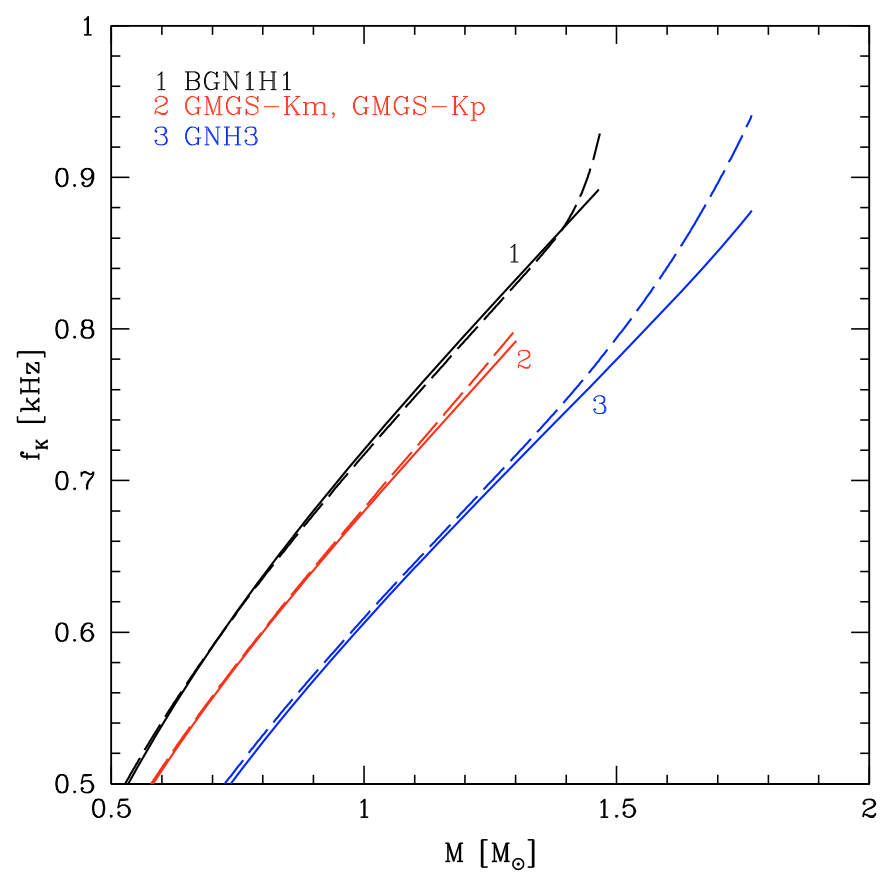

Fig. 2. (Color online) Same as Fig. 1 but for BGN1H1, GNH3, GMGS$\mathrm{Km}$ and GMGS-Kp EOSs. Notice that due to a very strong softening by the kaon condensate and the simultaneous constraint $M<0.9 M_{\max }^{\text {stat }}$, the GMGS-Km and GMGS-Kp curves do not contain kaon-condensed segments. Therefore, the curves for these EOSs coincide.

in Table 2. Strange stars are likely to have a thin normal matter crust, with bottom density, $\rho_{\mathrm{b}}$, not exceeding the neutron drip density $\rho_{\mathrm{ND}} \approx 4 \times 10^{11} \mathrm{~g} \mathrm{~cm}^{-3}$. The maximum mass of normal crusts is reached for $\rho_{\mathrm{b}}=\rho_{\mathrm{ND}}$.

First we consider strange stars with a maximum crust. We computed the coefficient $C_{\mathrm{SS}}=1.15 \mathrm{kHz}$ using an identical procedure to that for neutron stars described in the previous section. 


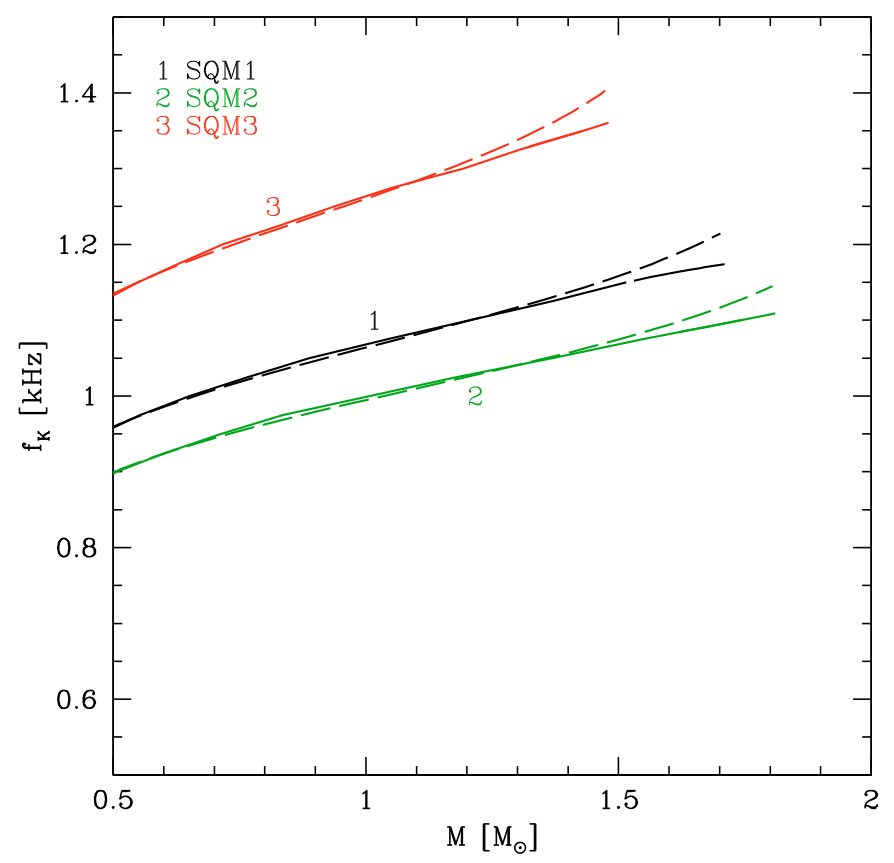

Fig. 3. (Color online) Same as Fig. 1 but for three EOSs of strange stars. Strange stars possess maximal crust, with bottom density $\rho_{\mathrm{b}}=\rho_{\mathrm{ND}}$. To obtain the dash lines, we used Eq. (6) with $C=C_{\mathrm{SS}}=1.15$.

Table 2. Parameters of the bag models for strange stars.

\begin{tabular}{ccccc}
\hline \hline EOS & $\begin{array}{c}B \\
{\left[\mathrm{MeV} \mathrm{fm}^{-3}\right]}\end{array}$ & $\begin{array}{c}m_{\mathrm{s}} c^{2} \\
{[\mathrm{MeV}]}\end{array}$ & $\begin{array}{c}M_{\max }^{\text {stat }} \\
{\left[M_{\odot}\right]}\end{array}$ & $\begin{array}{c}R_{1.4} \\
{[\mathrm{~km}]}\end{array}$ \\
\hline SQM1 & 56 & 200 & 1.90 & 11.27 \\
SQM2 & 45 & 185 & 2.02 & 11.86 \\
SQM3 & 67 & 205 & 1.65 & 9.94 \\
\hline
\end{tabular}

$B$ - MIT bag constant, $m_{\mathrm{s}}-$ strange quark mass. For all models, the QCD coupling constant equals $\alpha_{\mathrm{s}}=0.2$. The maximum allowable mass for non-rotating stars, $M_{\max }^{\text {stat }}$, and the circumferential radius of non-rotating stars of $1.4 M_{\odot}, R_{1.4}$, are given in the last two columns, respectively.

As we see in Fig. 3, the precision of an empirical formula within the mass range $0.5 M_{\odot}<M<0.9 M_{\max }^{\text {stat }}$ is as high as for neutron stars (typical relative deviation within $2 \%$, largest deviation of about $4 \%$ at highest masses). However, the value of $C$ is higher than for neutron stars.

Let us consider now quark star models with less massive crusts. These models were constructed assuming $\rho_{\mathrm{b}}<\rho_{\mathrm{ND}}$. The effect on the optimum value $C_{\mathrm{SS}} \approx$ $f_{\mathrm{K}}(M)\left(M / M_{\odot}\right)^{-1 / 2}(R / 10 \mathrm{~km})^{3 / 2}$ turned out to be very small. At a fixed $M$, a decrease of $\rho_{\mathrm{b}}$ leads to an increase of $f_{\mathrm{K}}$ (a more compact star). Simultaneously, however, the static value of $R(M)$ decreases, and therefore both effects cancel out to a large extent. Consequently, $C_{\mathrm{SS}}$ depends rather weakly on the crust mass, and one may use $C_{\mathrm{SS}}=1.15 \mathrm{kHz}$ for any crust size.

If strange quark matter is not the ground state of the matter at zero pressure, strange stars bound by the strong interactions cannot exist. However, one can still consider hybrid stars bound by gravitation, with quark matter cores inside a baryon envelope. Rapid rotation of hybrid stars with quark cores was studied by Ippolito et al. (2008). The transition to quark matter usually strongly softens the EOS, and decreases the values of $M_{\max }^{\text {rot }}$ and $M_{\max }^{\text {stat }}$. However, a quark core does not appear below $0.9 M_{\max }^{\text {stat }}$ and therefore does not appreciably influence the

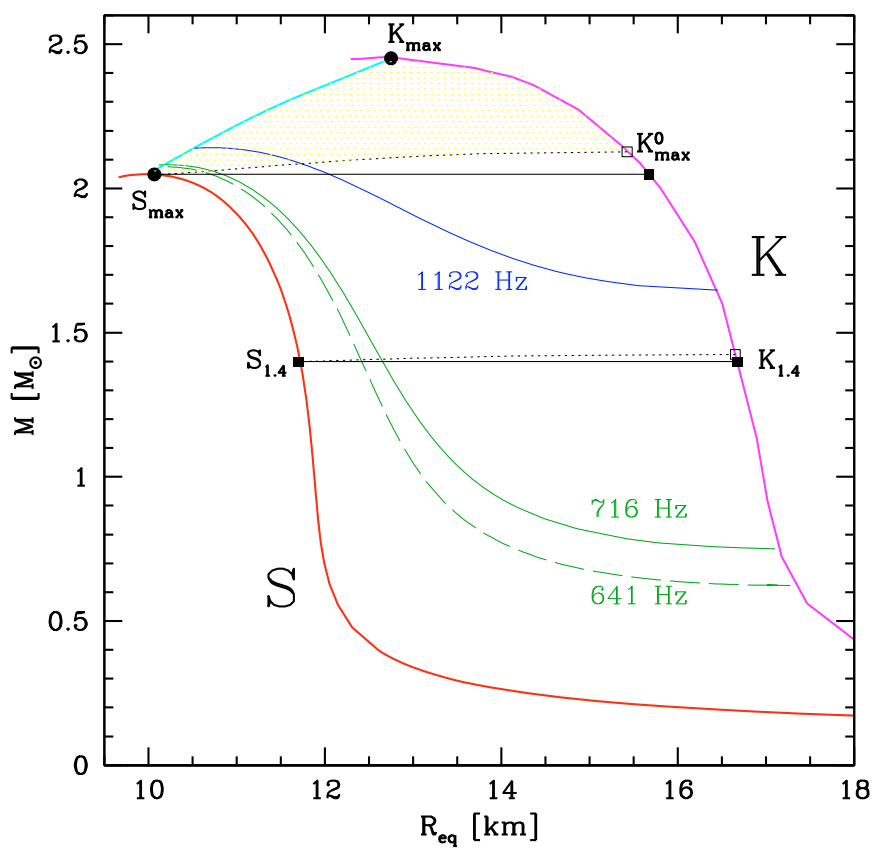

Fig. 4. (Color online) Gravitational mass, $M$, versus equatorial radius, $R_{\text {eq }}$, for static and rigidly rotating neutron stars, based on the DH EOS. Solid line $\mathbf{S}$ : static models (i.e., $R(M)$ ). Solid line K: Keplerian (massshedding) configurations (i.e., $R_{\mathrm{K}}(M)$ ). The area, bounded by the $\mathbf{S}$, $\mathbf{K}$ curves and a dash line $S_{\max } K_{\max }$, consists of points corresponding to stationary rotating configurations. Configurations belonging to a shaded triangular area above the dotted line $\mathrm{S}_{\max }-\mathrm{K}_{\max }^{0}$ have baryon mass $M_{\mathrm{b}}$ higher than the maximum allowable baryon mass for non-rotating stars, $M_{\mathrm{b} \text { max }}^{\text {stat }}$. Three lines corresponding to neutron stars rotating stably at $f \stackrel{\text { max }}{=} 641 \mathrm{~Hz}, 716 \mathrm{~Hz}$, and $1122 \mathrm{~Hz}$ are labeled with rotation frequencies. The nearly horizontal dotted line $\mathrm{S}_{1.4}-\mathrm{K}_{1.4}$ corresponds to configurations with fixed baryon number equal to that of the non-rotating star of gravitational mass $M=1.4 M_{\odot}$. Generally, solid lines connecting filled circles with filled squares correspond to $M=$ const., while dotted lines connecting filled circles with open squares correspond to stars with $M_{\mathrm{b}}=$ const. For further explanations see the text.

value of $C$, which is essentially identical to $C_{\mathrm{NS}}$. This situation is qualitatively similar to the case of kaon condensation, appearing in the GMGS-Km and GMGS-Kp EOSs.

\section{Static and rotating configurations in the mass-radius plane}

The formulae for $f_{\mathrm{K}}(M)$ are based on a one-to-one correspondence between a static configuration $\mathrm{S}$, belonging to static boundary $\mathbf{S}$ of the region of rotating configurations, and the rotation frequency of a Keplerian configuration $\mathrm{K}$ on the $\mathbf{K}$ boundary. This correspondence is visualized in Fig. 4, based on the numerical results obtained for the DH EOS. The frequency of rotation of a Keplerian configuration $\mathrm{K}$ is obtained via the mass and radius of a static configuration $S$ with same $M$. Both configurations are connected by a horizontal line in the $R_{\text {eq }}-M$ plane.

The empirical formula for the absolute upper bound on $f$ of stably rotating configurations for a given EOS, $f_{\max }^{\mathrm{EOS}}$ (Haensel \& Zdunik 1989; Friedman et al. 1989; Shapiro et al. 1989; Lattimer et al. 1990; Haensel et al. 1995), is of a different character. It results from an (approximate but precise) one-to-one correspondence between the parameters of two extremal 
configurations, static $S_{\max }$ and Keplerian $\mathrm{K}_{\max }$ (filled circles), and reads

$f_{\max }^{\mathrm{EOS}} \approx C\left(\frac{M_{\mathrm{max}}^{\mathrm{stat}}}{M_{\odot}}\right)^{1 / 2}\left(\frac{R_{M_{\mathrm{max}}^{\mathrm{stat}}}^{\mathrm{stat}}}{10 \mathrm{~km}}\right)^{-3 / 2}$,

where $C$ is to a very good approximation independent of the EOS. We have $C_{\mathrm{NS}} \approx C_{\mathrm{SS}}=1.22 \mathrm{kHz}$ (Haensel et al. 1995). This value is noticeably higher than $C_{\mathrm{NS}}$ or $C_{\mathrm{SS}}$, which determine $f_{\mathrm{K}}(M)$ for $0.5 M_{\odot}<M<0.9 M_{\max }^{\text {stat }}$.

The functional form of Eq. (7) is, in fact, exact in general relativity for uniform rotation of stars with the so-called minimum period EOS of Koranda et al. (1997). This EOS contains the single parameter $\epsilon_{\mathrm{c}}$, which is the transition energy density between the low-density EOS with $P=0$ and the high-density EOS with $P=\epsilon-\epsilon_{\mathrm{c}}$. The value of $C$ for the maximum mass case is $1.35 \mathrm{kHz}$.

In Fig. 4 we give the correspondence between the stellar configurations of the same baryon number. The line $S_{\max } \rightarrow$ $\mathrm{K}_{\max }^{0}$ separates "supramassive" configurations from the "normal" ones, which can be reached by spinning up a non-rotating star. The maximum rotational frequency for the "normal" sequences (reached at point $\mathrm{K}_{\max }^{0}$ ) has been discussed by Cook et al. (1994a,b) for polytropic and realistic EOSs (their Tables 3 and 7 respectively). It should be noted that this value cannot be estimated using our formula for $f_{\mathrm{K}}(M)$, Eq. (6), because our formula is valid within a restricted mass range $0.5 M_{\odot}<M<$ $0.9 M_{\text {max }}^{\text {stat }}$.

Formula (6) connects configurations of the same gravitational mass $M$. For example, it connects a $M=1.4 M_{\odot}$ nonrotating star (filled circle $-\mathrm{S}_{1.4}$ ) with a Keplerian configuration (filled square $-\mathrm{K}_{1.4}$ ), in Fig. 5. They are joined by a solid horizontal line. At a fixed baryon mass, $M_{\mathrm{b}}, M$ increases with increasing rotation frequency. The dotted line connecting the filled circle $\left(\mathrm{S}_{1.4}\right)$ and the open square near $\mathrm{K}_{1.4}$ contains configurations with fixed $M_{\mathrm{b}}$, equal to that of a non-rotating star with $1.4 M_{\odot}$. Deviation of the solid line from the dotted one shows the rotational increase of $M$ at a fixed $M_{\mathrm{b}}$. For a $1.4 M_{\odot}$ star, the fractional increase is equal to $1.7 \%$, and for the maximum static mass (dotted line $\mathrm{S}_{\max } \rightarrow \mathrm{K}^{0}{ }_{\text {max }}$ ) it reaches $3.8 \%$.

\section{Relation between $R(M)$ and $R_{K}(M)$}

Let us consider a family (sequence) of stationary configurations rotating stably at a frequency $f$. They form a curve in the $M-R_{\text {eq }}$ plane (see examples in Fig. 4). The curve is bound at $R_{\text {eq }}=R_{\min }(f)$ by the axisymmetric instability, implying star collapse into a Kerr black hole. The largest circumferential radius is reached for the Keplerian configuration, $R_{\max }(f)=R_{\mathrm{K}}(M)$. Bejger et al. (2007) have shown that $R_{\max }(f)$ is (within $2 \%$ ) equal to the radius of an orbit of a point particle moving in the Schwarzschild space-time around a point (or a spherical) mass $M$. This implies

$R_{\max }(f) \approx\left(\frac{G M}{4 \pi^{2} f^{2}}\right)^{1 / 3}$.

For convenience, we introduce a frequency $f_{0}$,

$f_{0}=\frac{1}{2 \pi} \sqrt{\frac{G M_{\odot}}{(10 \mathrm{~km})^{3}}}=1.8335 \mathrm{kHz}$.

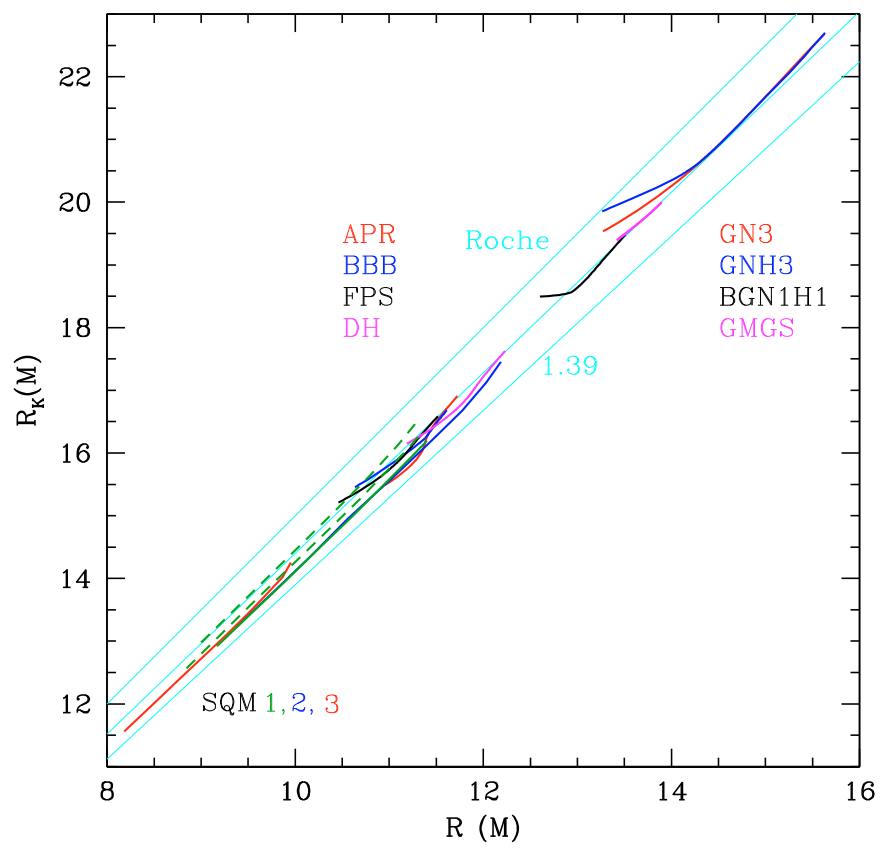

Fig. 5. (Color online) Equatorial circumferential radius of the Keplerian configuration, $R_{\mathrm{K}}(M)$, vs. circumferential radius of the static configuration $R$ of the same gravitational mass $M$, for $0.5 M_{\odot}<M<0.9 M_{\max }^{\text {stat }}$, for neutron stars (solid lines) and quark stars with crust (dash lines). The three straight cyan lines are: upper line $a_{\text {Roche }}=1.5$, middle line $a=a_{\mathrm{NS}}=1.44$, and bottom line $a=1.39$. Neutron stars: The color of the curve for a given EOS coincides with that of the EOS label (APR, ..., DH). Strange stars: nearly straight green, blue, and red solid lines, located in the lower bundle, correspond to the SQM1, SQM2, and SQM3 EOSs of Table 2 with a maximum solid crust. Green dashed lines in this bundle describe results obtained for the SQM1 EOS of the quark core and low-mass crusts: $0.3 M_{\mathrm{cr}, \max }$ (middle green line) and $0.06 M_{\mathrm{cr}, \max }$ (upper green line).

The validity of the empirical formula, Eq. (6), then suggests an approximate proportionality

$R_{\mathrm{K}}(M) \approx a R(M), a=\left(\frac{C}{f_{0}}\right)^{-2 / 3}$

For the extreme relativistic Roche model, $R_{\mathrm{K}}(M)$ is strictly proportional to $R(M)$, and $a_{\text {Roche }}=1.5$, Eq. (4). For neutron stars and quark stars with crusts, with masses within $0.5 M_{\odot}<M<$ $0.9 M_{\max }^{\text {stat }}$, the proportionality holds within a few percent, as shown in Fig. 5. However, the best-fit proportionality factors are smaller than 1.5 of the Roche model, $a_{\mathrm{NS}} \approx a_{\mathrm{SS}} \approx 1.44$.

The dependence of $a_{\mathrm{SS}}$ on the crust mass, $M_{\mathrm{cr}}$, is very weak. This can be explained via the effects of $M_{\mathrm{cr}}$ on $R(M)$ and $f_{\mathrm{K}}(M)$. These effects are opposite: at fixed $M, R(M)$ increases, and $f_{\mathrm{K}}(M)$ decreases, with increasing $M_{\mathrm{cr}}$. The cancellation of both effects results in an only slight decrease of $a_{\mathrm{SS}}$ with increasing $M_{\text {cr }}$ (see Fig. 5).

Lasota et al. (1996) derived an approximate relation between equatorial radius of a maximally rotating configuration, $R_{f_{\max }}^{\text {rot }}$ and the radius of non-rotating neutron star with maximum allowable mass, $R_{M_{\max }}^{\text {stat }}$. The maximally rotating configuration, stable both with respect to mass shedding and axisymmetric perturbations, is actually very close to that with the highest mass, $M_{\max }^{\text {rot }}$ (in Fig. 4 they are indistinguishable). The approximate proportionality found by Lasota et al. (1996) for neutron stars is $R_{f_{\max }}^{\text {rot }} \approx$ $1.32 R_{M_{\max }}^{\text {stat }}$. This relation connects two extremal configurations. They have different masses, related by $M_{\max }^{\text {rot }} \approx 1.18 M_{\max }^{\text {stat }}$ 
(Lasota et al. 1996). In contrast, Eq. (10) connects normal configurations of neutron stars and strange stars with the same gravitational mass and holds for $0.5 M_{\odot}<M<0.9 M_{\max }^{\text {stat }}$.

\section{Discussion and conclusions}

We have tested empirical formulae for the Keplerian (mass shedding) frequency of neutron stars of mass $M$, proposed by Lattimer \& Prakash (2004). Using numerical results of precise 2D calculations, performed for ten representative realistic EOSs of dense matter based on different dense matter models, we find the prefactor $C_{\mathrm{NS}}=1.08 \mathrm{kHz}$, slightly higher than $1.04 \mathrm{kHz}$ proposed by Lattimer \& Prakash (2004). With our prefactor, the formula is precise for $0.5 M_{\odot}<M<0.9 M_{\max }^{\text {stat }}$ (typically within $2 \%$, maximum deviation occurring for highest $M$ not exceeding $5 \%$ ). Strange stars can reach larger $f_{\mathrm{K}}(M)$ than neutron stars. With a maximum crust on strange stars, we get $C_{\mathrm{SS}}=1.15 \mathrm{kHz}$. The value of $C_{\mathrm{SS}}$ does not depend significantly on the crust mass, and also can be used for bare strange stars. We notice that both $C_{\mathrm{NS}}$ and $C_{\mathrm{SS}}$ are significantly larger than for the relativistic Roche model, $C_{\text {Roche }}=1.00 \mathrm{kHz}$ (Shapiro et al. 1983).

Using an approximate but quite precise Schwarzschild-like formula, relating $M, R_{\mathrm{K}}$, and $f_{\mathrm{K}}$ (Bejger et al. 2007), we show that to a very good approximation the mass-shedding radius at a given $M$ is proportional to the static radius $R(M)$, provided $0.5 M_{\odot}<M<0.9 M_{\max }^{\text {stat }}$. For neutron stars and strange stars we obtain the best-fit proportionality factor $a_{\mathrm{NS}} \approx a_{\mathrm{SS}} \approx 1.44$. These proportionality factors are smaller than the exact factor $1.5 \mathrm{ob}-$ tained for the relativistic Roche model.

We derived a set of empirical formulae, expressing Keplerian frequency and equatorial radius of the Keplerian configuration in terms of the mass and radius of the normal configuration of the same mass. These formulae can be used for masses $0.5 M_{\odot}<$ $M<0.9 M_{\max }^{\text {stat }}$. The formulae are approximate but quite precise, and therefore might be useful constraining the EOS of dense matter by the observations of pulsars.

Acknowledgements. This work was partially supported by the Polish MNiSW grant No. N20300632/0450 and by the US DOE grant DE-AC02-87ER40317.
M.B. was partially supported by Marie Curie Fellowship No. ERG-2007-224793 within the 7th European Community Framework Programme.

\section{References}

Akmal, A., Pandharipande, V. R., \& Ravenhall, D. G. 1998, Phys. Rev. C, 58, 1804

Backer, D. C., Kulkarni, S. R., Heiles, C., et al. 1982, Nature, 300, 61

Balberg, S., \& Gal, A. 1997, Nucl. Phys. A., 625, 435

Balberg, S., Lichtenstadt, I., \& Cook, G. B. 1999, ApJ, 121, 515

Baldo, M., Bombaci, I., \& Burgio, G. F. 1997, A\&A, 328, 274

Bejger, M., Haensel, P., \& Zdunik, J. L. 2007, A\&A, 464, L49

Bonazzola, S., \& Gourgoulhon, E. 1994, Class. Quantum Grav., 11, 1775

Bonazzola, S., Gourgoulhon, E., Salgado, M., \& Marck, J.-A. 1993, A\&A, 278, 421

Bonazzola, S., Gourgoulhon, E., \& Marck, J.-A. 1998, Phys. Rev. D, 58, 104020 Cook, G. B., Shapiro, S. L., \& Teukolsky, S. A. 1994a, ApJ, 422, 227 Cook, G. B., Shapiro, S. L., \& Teukolsky, S. A. 1999, ApJ, 424, 823 Douchin, F., \& Haensel, P. 2001, A\&A, 380, 151

Farhi, E., \& Jaffe, R. L. 1984, Phys. Rev. D, 30, 2379

Friedman, J. L., Ipser, J. R., \& Parker, L. 1989, Phys. Rev. Lett., 62, 3015

Glendenning, N. K. 1985, ApJ, 293, 470

Gourgoulhon, E., \& Bonazzola, S. 1994, Class. Quantum Grav., 11, 443

Gourgoulhon, E., Haensel, P., Livine, R., et al. 1999, A\&A, 349, 851

Haensel, P., \& Zdunik, J. L. 1989, Nature, 340, 313

Haensel, P., Salgado, M., \& Bonazzola, S. 1995, A\&A, 296, 745

Haensel, P., Potekhin, A. Y., \& Yakovlev, D. G. 2007, Neutron Stars 1, Equation of State and Structure (New York: Springer)

Hessels, J. W. T., Ransom, S. M., Stairs I. H., et al. 2006, Science, 311, 1901

Ippolito, N. D., Ruggieri, M., Rischke, D. H., Sedrakian, A., \& Weber, F. 2008, Phys. Rev. D, 77, 023004

Kaaret, P., Prieskorn, Z., In't Zand, J. J. M., et al. 2007, ApJ, 657, L97

Koranda, S., Stergioulas, N., \& Friedman, J. L. 1997, ApJ, 488, 799

Lasota, J.-P., Haensel, P., \& Abramowicz, M. A. 1996, ApJ, 456, 426

Lattimer, J. M., \& Prakash, M. 2004, Science, 304, 536

Lattimer, J. M., \& Prakash, M. 2006, Nucl. Phys. A, 777, 479

Lattimer, J. M., Prakash, M., Masak, D., \& Yahil, A. 1990, ApJ, 355, L241

Lorenz, C. P., Ravenhall, D. G., \& Pethick, C. J. 1993, Phys. Rev. Lett., 70, 379

Pandharipande, V. R., \& Ravenhall, D. G. 1989, in Proc. NATO Advanced Research Workshop on nuclear matter and heavy ion collisions, Les Houches, ed. M. Soyeur et al. (New York: Plenum), 103

Pons, J. A., Reddy, S., Ellis, P. J., Prakash, M., \& Lattimer, J. M. 2000, Phys. Rev. C, 62, 035803

Shapiro, S. L., \& Teukolsky, S. A. 1983, Black Holes, White Dwarfs, and Neutron Stars (New York: Wiley)

Shapiro, S. L., Teukolsky, S. A., \& Wasserman, I. 1983, ApJ, 272, 702

Shapiro, S. L., Teukolsky, S. A., \& Wasserman, I. 1989, Nature, 340, 451

Zdunik, J. L. 2000, A\&A, 359, 311 